\title{
An Optimizing Approach for Multi Constraints Reassignment Problem of Human Resources
}

\author{
Said Tkatek ${ }^{1}$, Otman Abdoun ${ }^{2}$, Jaafar Abouchabaka ${ }^{1}$, Najat Rafalia ${ }^{1}$ \\ ${ }^{1}$ LaRIT, Faculty of Sciences, IbnTofail University Kenitra, Morocco \\ ${ }^{2}$ Pluridisciplinary Laboratory, Polydisciplinary Faculty, Abdelmalk Essaadi University,Larache, Morocco
}

\begin{tabular}{l}
\hline Article Info \\
\hline Article history: \\
Received Nov 16, 2015 \\
Revised Apr 4, 2016 \\
Accepted Apr 27, 2016 \\
\hline
\end{tabular}

Keyword:

Genetic algorithm

Multi constraints

Productivity

Reassignment problem

\begin{abstract}
This paper presents an effective approach to optimize the reassignment of Human Resources in the enterprise that is formed by several units of productions to take into consideration the human characteristics. This approach consists of two steps; the first step is to formalize the studied problem that is practically take the form of the generalized assignment problem (GAP) known as NP-hard problem. Additionally, the variables in the formulation of our problem are interlinked by certain constraints. These two proprieties can to justify the important complexity of this problem. The second step is focused to solve this complex problem by using the genetic algorithm. We present the experimentally result for justifying the validity of the proposed approach. So, the solution obtained allowed us to get an optimal assignment of personnel that can lead to improve the average productivity or ratability or at least ensure its equilibration within sites of enterprise.
\end{abstract}

Copyright $(2016$ Institute of Advanced Engineering and Science. All rights reserved.

\section{Corresponding Author:}

Otman Abdoun,

Departement of Computer Science,

Polydisciplinary Faculty, Abdelmalek Essaadi University,

B.P 745, Main Post 92004 - Larache - Morocco.

Email: abdoun@fpl.ma

\section{INTRODUCTION}

The human resources can be seen as a source of sustained competitive advantage for organizations and a major asset in their strategic reorientation. Furthermore, for a more dynamic management of these resources, the managers of the organizations of production attempt to identify suitable profiles to maintain and develop their productivity. Given that the theory based on human resources show that the good productivity in the enterprises can be resulted in the way how the assignment of human resources is performed [1]-[2]. The personnel reassignment can be considered as an important factor to dispose the human resources quality that can be capable to contribute for meeting the desired objective by the manager and its serves to reduce the recruitment costs. In the large enterprises or administrations decomposed by several production units and distributed across multiple geographical sites, this reassignment processes or processes mobility can to lead to improve or to maintain the productivity within the production units and to motivate the agents.

Generally, each agent occupy a post in the original site and want to change this site another voluntarily or by redeployment, is identified by individual weight calculated by basing socio-professional criteria function. This weight can be grouped the competency, the profitability and the individual performance ...etc. In order to optimize the best repartition of personnel and to reaching the objectives fixed, the managers must to dispose an optimizing tools based on a formal model by integrating these specific weights and mobility constraints [3].

The work [4] addresses a theoretical model based for empirical facts on repartition of workers across clusters with different labor productivity. Its key idea is to assume that there are restrictions on 
capacity of workers for clusters with high productivity. The work [5] is focused to find a solution for assignment problem by respecting the different constraints relative to labor regulations and a constraint relative to multiple sites, balance the workload over employees but not addressing in the problem the impact these displacements of human resources on the productivity in the sites and not taking into account the constraints related to cyclic assignment and conservation of posts.

Generally, Our work is the complements of our previous works [1]-[3], is focused on diversity of possible forms and relationship between the reassignment which also we label the mobility of human resources, and his positively impact on the productivity in the enterprise basing on the specific weight by respecting of several constraint. The formulation developed in this work illustrate that this problem can be classified as constrained combinatorial optimization problems and can be taken a generalized assignment problem form known as NP-hard problem [6] and its practically similar to multi index assignment problems (MIAPs) [7].

To resolve this problem, we are interested to implement the genetic algorithms which can be characterized by an efficiency to solve NP-hard problems. Additionally, the optimal solution obtained must be ensured the improvement the average productivity for each unit of production through the right choice of parameters of this problem. For this, For this, the genetic operators used for starting the genetic algorithm are uniform selection, operator Matrix crossing $U X$ (Uniform Crossover) [8], Operator Matrix mutation HPRM that is combined between uniform mutation [9] and HPRM operator [10]. We call again that the operators $U M$ and HPRM have provided encouraging results in solving the traveling salesman problem (TSP) which is an NP-complete problem [10].

This paper is organized as follows: firstly we present the mathematical formalism modeling this approach and evaluate its complexity. Secondly we will implement genetic algorithms to find the optimal repartition of human resources to improving the average productivity and to satisfying it. Computational results obtained on randomly generated instances are reported to evaluate the validity approach.

\section{FORMULATION OF THE PROBLEM}

\subsection{Representation of the Problem}

This new work is considered as the complement the work presented in paper [1]-[2]. In addition, we consider that the enterprise is decomposed into several production units $E_{j}$ with $j \in[1, N U]$ geographically distributed. The number of employee $\widetilde{N}_{j k}$ wishing to displace from a unit $E_{j}$ to another $E_{k}$ is symbolized by $E_{j k}^{\mathrm{i}}$ and is possessed the specific weight $W_{j k}^{i}, i \in\left[1, \widetilde{N}_{j k}\right]$. The set of this group is:

$$
E_{j k}=\left\{E_{j k}^{1}, E_{j k}^{2}, E_{j k}^{\mathrm{i}}, \ldots, E_{j k}^{N_{J k}}\right\}
$$

Ket:

- $\widetilde{N}_{j}=\sum_{k=1}^{N E} \widetilde{N_{j k}}$ is the number of the candidate's employees to reassign from $E_{j}$ to $E_{k}$. $(\forall j \in[1, N U])$.

- $X_{j k}^{i}$ is the bivalent variables, the employee is reassigned from one unit $E_{j}$ to another $E_{k}$ if $X_{j k}^{i}=1$ and 0 otherwise.

Initially, the all candidates wishing to mobilize must be verified the objective constraint given by:

$$
\bar{W}_{k} \geq \alpha_{k} \bar{W}_{k}^{0}
$$

- $\alpha_{\mathrm{k}}$ is the tolerance coefficient parametrizing this constraint .

- $\overline{\mathrm{W}}_{\mathrm{k}}$ is the average weight engendered by candidates moved to the unit $E_{k}$.

- $\overline{\mathrm{W}}_{\mathrm{k}}^{0}$ is the average weight of candidates initially worked in the unit $E_{k}$.

For the problem studied, we can list several constraints:

\subsection{Cost constraint}

We assume that the posts can be occupied by an agent $\left(X_{k j}^{i}=1\right)$ which consumes a variety of the enterprise resources $r_{i j}^{k}$. The global consumed resources is limited by the capacity resource $R_{k}$. The expression of resource constraint as follows:

$$
\sum_{\substack{j=1 \\ j \neq k}}^{N U} \sum_{i=1}^{\widetilde{N}_{j k}} r_{i j}^{k,} X_{i j k}^{k} \leq R_{k}
$$




\subsection{Equilibrium constraint of posts}

This constraint explain that the number of occupied posts by the candidates reallocated from department $E_{j}$ to unit $E_{k}$ must be equal the number of vacated posts by candidates which leave the same unit. The expression of this constraint is expressed by:

$$
\sum_{\substack{j=1 \\ j \neq k}}^{N U} \sum_{i=1}^{\widetilde{N}_{j k}} X_{j k}^{i}=\sum_{\substack{k=1 \\ j \neq k}}^{N U} \sum_{i=1}^{\widetilde{N}_{k}} X_{k j}^{i}
$$

\subsection{Capacity constraint}

The capacity constraint requires which the number $N_{k}$ of agent reassigned to the department $E_{k}$ is limited by an $C_{k}$ imposed by the managers; the expression is given as follows:

$$
\sum_{j=1}^{N S} \sum_{i=1}^{\widetilde{N}_{j k}} X_{j k}^{i} \leq C_{k}
$$

when the resources of the posts are identical, the resource constraint can be reduced capacity.

\subsection{Priority constraint}

These weights are the elements of the sub-matrix $\beta^{k, \alpha_{k}}$ :

$$
\begin{aligned}
& \text { if } W_{j k}^{i} \leq W_{j^{\prime} k}^{i} \text { and } X_{j k}^{i}=1 \text { then } X_{j \prime k}^{i}=1 ; \\
& i \leq l ; 1 \leq i \leq \max \left(\widetilde{N}_{k}\right) ; j \neq k
\end{aligned}
$$

Or:

$$
X_{j k}^{l}\left(\sum_{i=1}^{l} X_{j k}^{i}-1\right)=0 \forall l \in\left[1, \widetilde{N}_{k}\right]
$$

\subsection{Uniqueness constraint}

Another constraint can be added to the other constraints that is the uniqueness constraint which explains a candidate can't have two posts simultaneously in two departments $E_{k}$ :

$$
\sum_{k=1}^{N E} X_{i j k} \leq 1 \forall i \in\left[1, \widetilde{N}_{j k}\right], \forall j \in[1, N E]
$$

Based on the work [1], the number of candidate reassigned to the unit of production $E_{k}$ can be given by:

$$
N_{k}=\sum_{\substack{j=1 \\ j \neq k}}^{N U} \sum_{i=1}^{\widetilde{N}_{j k}} X_{j k}^{i}
$$

The global weight engendered by the assigned agents is given by:

$$
W_{k}^{\text {global }}=\sum_{\substack{j=1 \\ j \neq k}}^{N U} \sum_{i=1}^{\widetilde{N}_{j k}} W_{j k}^{i} X_{j k}^{i}
$$

The average weight associated with $N_{k}$ assigned agents is given by:

$$
\bar{W}_{k}=\frac{W_{k}^{\text {global }}}{N_{k}}
$$

Let :

$$
\bar{W}_{k}=\frac{1}{N_{k}} \sum_{\substack{j=1 \\ j \neq k}}^{N U} \sum_{i=1}^{\widetilde{N}_{j k}} W_{j k}^{i}
$$

The combination of equation (1) and (9) is given by: 


$$
\sum_{\substack{j=1 \\ j \neq k}}^{N U} \sum_{i=1}^{\widetilde{N}_{j k}}\left(W_{j k}^{i} X_{j k}^{i}-\alpha_{k} \bar{W}_{k}^{0} X_{j k}^{i}\right) \geq 0
$$

If we put: $\beta_{j k}^{i \alpha_{k}}=\left(W_{j k}^{i}-\alpha_{k} \bar{W}_{k}^{0}\right)$, we will construct a sub matrix assignment noted $\beta^{k, \alpha}$ or:

$$
\beta_{k}^{\alpha_{k}}=\left\{\beta_{j i k}^{\alpha_{k}} / 1 \leq j \leq N U ; 1 \leq i \leq \max \left(\widetilde{N}_{j k}\right) ; j \neq k\right\}
$$

Similarly, we construct a sub-matrix $X_{k}$ such that:

$$
X^{k}=\left\{X_{j k}^{i, \alpha_{k}} / 1 \leq i \leq N U ; 1 \leq j \leq \max \left(\widetilde{N}_{j k}\right) ; j \neq k\right\}
$$

We assume that the tolerance coefficient $\alpha_{k}$ associated with unit production $E_{k}$ are equals. So, the global formulation of this problem is written as follows:

$$
\operatorname{Max}\left(F^{a}\right)=\operatorname{Max} \sum_{k=1}^{N U} \operatorname{Trace}\left(\beta_{k}^{\alpha_{k}} X_{k}\right)
$$

Under the constraints:

$$
\left\{\begin{array}{lc}
\operatorname{Trace}\left(\beta_{k}^{\alpha_{k}} X_{k}\right) \geq 0 \forall & k \in[1, N U] \\
X_{j k}^{i}\left(\sum_{i=1}^{l} X_{j k}^{i}-l\right)=0 & \forall l \in\left[1, \widetilde{N}_{j k}\right] \\
\sum_{j=1}^{N U} \sum_{i=1}^{\widetilde{N}_{j k}} X_{i j}^{k} \leq C_{k} & \forall k \in[1, N U] \\
\sum_{j \neq k}^{N U} \sum_{i=1}^{\widetilde{N}_{j k}} r_{j k}^{i} X_{j k}^{i} \leq R_{k} & \forall k \in[1, N U] \\
\sum_{k=1}^{N U} X_{j k}^{i} \leq 1 \quad \forall i \in\left[1, \widetilde{N}_{j k}\right], \forall j \in[1, N U]
\end{array}\right.
$$

The weight generated by $N_{k}$ candidates reassigned to the unit $E_{k}$ is:

$$
W_{k}^{+}=\sum_{\substack{j=1 \\ j \neq k}}^{N U} \sum_{i=1}^{\widetilde{N}_{j k}} W_{j k}^{i} X_{j k}^{i}
$$

The weight generated by the same number $N_{k}$ of candidates moved from the unit $E_{k}$ is:

$$
W_{k}^{-}=\sum_{\substack{j=1 \\ j \neq k}}^{N U} \sum_{i=1}^{\widetilde{N}_{j k}} W_{k j}^{i} X_{k j}^{i}
$$

The improvement weight by the reassignment processes associated to the unit $E_{k}$ is:

$$
\begin{array}{ll}
\Delta W_{k}=W_{k}^{+}-W_{k}^{-} \\
\Delta W_{k}=\left(\sum_{i=1}^{N_{k}} W_{k}^{i}\right)_{\text {in }}-\left(\sum_{i=1}^{N_{k}} W_{k}^{i}\right)_{\text {out }} &
\end{array}
$$

Generally, within unit of production, this gap expresses the improving global weight if $\Delta \boldsymbol{W}_{\boldsymbol{k}}>0$ and he explain the equilibrium of this global weight if $\Delta \boldsymbol{W}_{\boldsymbol{k}}=\mathbf{0}$.

\section{COMPLEXITY OF THE PROBLEM}

The assignments of candidates to the units of production are linked. In addition, the formulation of the problem is practically similar to the mathematical model for the Generalized Assignment Problem (GAP) that it's NP-hard [11] and can be considered such as multi index assignment problems (MIAPs).

The generalized assignment problem (GAP) examines the minimum cost assignment of $\mathrm{n}$ jobs to $\mathrm{m}$ agents such that each job is assigned to exactly one agent subject to capacity restrictions on the agents. The generalized assignment problem is an NP-hard combinatorial optimization problem [12]. The formulation of this problem is: 
$(\mathrm{GAP}):\left\{\begin{array}{l}\operatorname{Max} \sum_{i} \sum_{j} c_{l j} x_{l j} \\ \sum_{j} a_{l j} x_{l j} \leq b_{i} \forall i \in I \\ \sum_{i} x_{i j}=1 \forall j \in J\end{array}\right.$

- $c_{i j}$ : the cost of assigning job $j$ to agent $I$;

- $a_{i j}$ : the capacity absorption when job $j$ is assigned to agent $i$;

- $b_{i}$ : the available capacity of agent $i$

- $x$ : the assignment variable equals 1 if agent $i$ is to perform job $j, 0$ otherwise.

This table summarizes the correspondence between GAP and MCRPHR :

Table 1. Correspondence of the complexity between (GAP) and (MCRPHR)

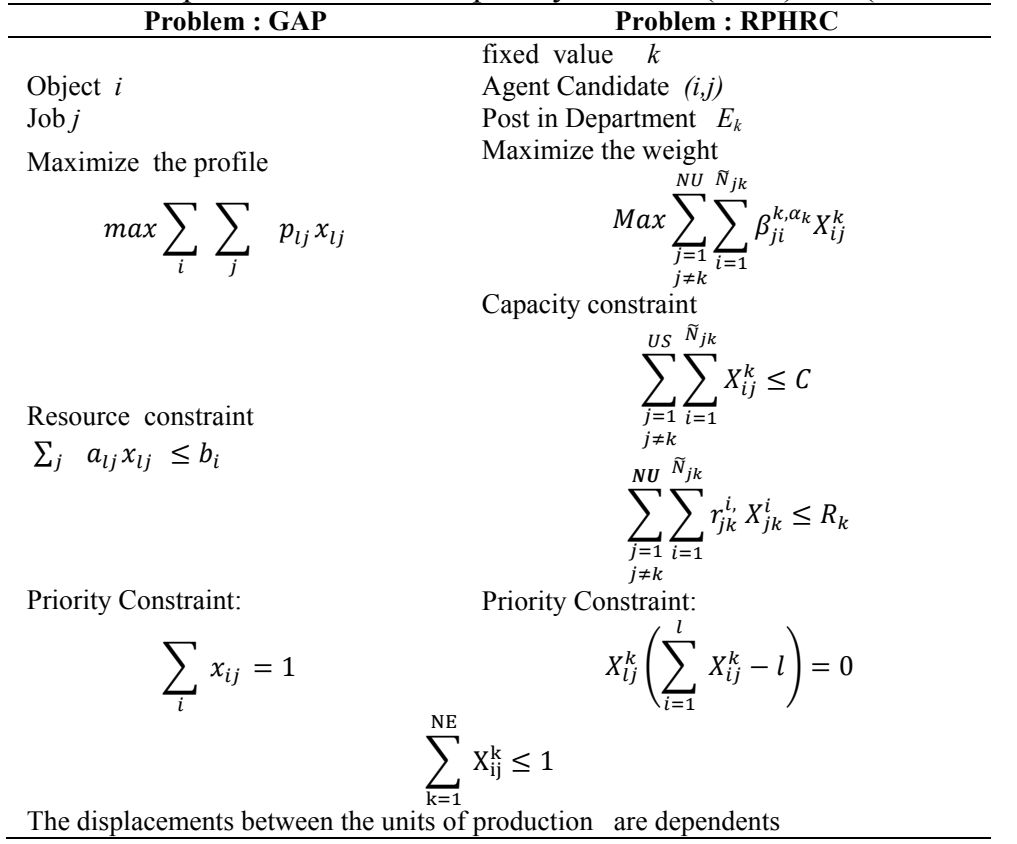

We note that the reallocation problem can be decomposed into several inter-related sub reallocation problem $\boldsymbol{P}_{\boldsymbol{k} \mathbf{0}}$ together by a variable $k$ and other constraint related to this variable such as the capacity constraint, positions Conservation of constraint and strain posts cost . A sub problem can be formulated as follow for $k=k_{0}$ :

$$
\operatorname{Max}\left(F^{a}\right)=\operatorname{Max}\left(\operatorname{Trace}\left(\beta_{k 0}^{\alpha} X_{k 0}\right)\right)
$$

Under the constraints: 


$$
\left(\boldsymbol{P}_{\boldsymbol{k} \mathbf{0}}\right):\left\{\begin{array}{l}
\operatorname{Trace}\left(\beta_{k 0}^{\alpha} X_{k 0}\right) \geq 0 \\
X_{j i}^{k 0}\left(\sum_{i=1}^{l} X_{j i}^{k 0}-l\right)=0, \forall l \in\left[1, \widetilde{N}_{j k 0}\right] \\
\sum_{j \neq k 0}^{N U} \sum_{i=1}^{\widetilde{N}_{j}} X_{i j}^{k 0} \leq C_{k 0}, \forall i \in\left[1, \widetilde{N}_{j k 0}\right] \text { and } \forall j \in[1, N U] \\
\sum_{j=1}^{N U} \sum_{i=1}^{\widetilde{N}_{j k 0}} r_{j i}^{k 0} X_{j i}^{k 0} \leq R_{k 0}, \forall i \in\left[1, \widetilde{N}_{j k 0}\right] \text { and } \forall j \in[1, N U] \\
\sum_{k=1}^{N U} X_{j k 0}^{i} \leq 1, \forall i \in\left[1, \widetilde{N}_{j k 0}\right] \text { and } \forall j \in[1, N U]
\end{array}\right.
$$

We notice that this formulation is similar to generalized assignment problem (GAP) known in the literature as a NP-hard problem [12]. For this, the complexity of the multi constraints reassignment problem can be evaluated and justified by the complexity of the (GAP).

For a given instance, our problem studied consist to search the optimal solutions allowing to maximize the objective function $F(X)$ by using a metaheuristic algorithm such as the genetic algorithm.

\section{GENETIC ALGORITHM OF RESOLUTION}

\subsection{Genetic algorithm}

The method used for solving this problem is the genetic algorithms. It is part of the family of evolutionary algorithms. They have attracted the interest of many researchers starting with Holland [13], who developed the basic principles through Goldberg [14], which used to solve real problems of optimization. Other researchers have followed this path including Davis [15], Mahfoud [16], Michalewicz [17], etc ...

In our approach, we used as a real encoding method of representation, the binary representation of sub-matrix, each sub matrix is composed by lines vectors that can be encoded by an array of Booleans: 0 or 1. The applied methods in this approach are:

- Random generation of the initial population;

- Uniform Selection (US);

- Matrix Crossover (MOX);

- Matrix Mutation (MMP);

- Insertion Method (inserting elitism).

\subsection{Global algorithm}

The global algorithm is composed of the following instructions: 


\section{STEP 1}

Define the tolerance coefficient $\alpha$

Define the number of units $N U$

Define the candidate's number in each unit $E_{j}$

Define the precision

Constructing the global matrix: $\beta^{\alpha}=\left\{\beta_{k}{ }^{\alpha} \quad / 1 \leq k \leq N S\right\}$

\section{STEP 2}

Generating the initial population composed of $N$ binary matrix assignment :

$$
X=\left\{X_{k} / 1 \leq k \leq N S\right\}
$$

\section{STEP 3}

Calculate $F^{\alpha}(X)=\sum_{k=1}^{N S} \quad \operatorname{Trace}\left(\beta_{k}^{\alpha_{k}} X_{k}\right)$ and check constraints,

Arranging the solutions in the list $N$

\section{STEP 4}

Iteration $=1$

\section{Repeat}

Select two solutions $\mathrm{X} 1$ and $\mathrm{X} 2$ from the list $\mathrm{N}$

If $\operatorname{Max}\left(F^{\alpha}(X C 1), F^{\alpha}(X C 2)\right)>\operatorname{Max}\left(F^{\alpha}(X 1), F^{\alpha}(X 2)\right)$ and check constraints Then

Cross $\mathrm{X} 1$ and $\mathrm{X} 2$ :

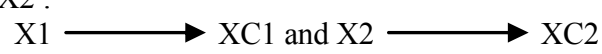

If $F^{\alpha}(X C 1)>F^{\alpha}(X C 2)$ then

Else

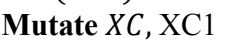

End if

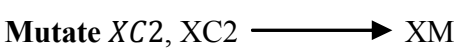

If $F(X M)>\operatorname{Max}(F(X C 1), F(X C 2))$ and $X M$ verifies the constraints then Insert the solution in the list $N$ Iteration $=$ Iteration +1

Else

End if

$$
\text { Reject } X M
$$

Until $\left(F^{\alpha}(X M)-F^{\alpha}(X 0)\right) / F^{\alpha}(X 0)<$ Precision

END

\section{EXPERIMENT}

\subsection{Description of the instance of the studied problem}

In this section, we present an example to valid this approach by using the genetic algorithm. For this, we consider an enterprise composed of three production units $(E 1, E 2$ and $E 3)$. Our work approach is based on the following hypotheses:

- Each unit includes a number of agents wanting to change their original post of work by taking into account their choices.

- The data associated with individual weights of candidates are randomly generated in the interval [50, $100]$.

- The all posts are possessed the same cost value $r_{j k}^{i}$.

- The tolerance coefficients value is identical for each unit of production: $\left(\alpha_{k=1}=\alpha_{k=2}=\alpha_{k=3}=\alpha\right)$.

The Table (2, 3 and 4) summarize the values of the individual's weight of candidates in each unit of production and for several of tolerance coefficient values $\alpha$. Moreover, the Table 5 summarize the constraint values $C_{k}$ and the average weight values $\bar{W}_{0}^{k}$. 


\begin{tabular}{cccccccc}
\multicolumn{1}{c}{ Table 2. Change of post to the unit E1 (k=1) } \\
\hline \multirow{2}{*}{ Original site } & $\begin{array}{c}\beta_{i j} \\
(\alpha=0)\end{array}$ & $\begin{array}{c}\beta_{i j} \\
(\alpha=1 / 4)\end{array}$ & $\begin{array}{c}\beta_{i j} \\
(\alpha=1 / 3)\end{array}$ & $\begin{array}{c}\beta_{i j} \\
(\alpha=1 / 2)\end{array}$ & $\begin{array}{c}\beta_{i j} \\
(\alpha=2 / 3)\end{array}$ & $\begin{array}{c}\beta_{i j} \\
(\alpha=3 / 4)\end{array}$ & $\begin{array}{c}\beta_{i j} \\
(\alpha=1)\end{array}$ \\
\hline E2 & $\mathbf{9 7}$ & 76 & 70,67 & 60 & 49,33 & 44 & 28 \\
E2 & $\mathbf{7 5}$ & 67 & 61,67 & 51 & 40,33 & 35 & 19 \\
E2 & $\mathbf{6 9}$ & 43 & 37,67 & 27 & 16,33 & 11 & -5 \\
E2 & $\mathbf{6 7}$ & 43 & 37,67 & 27 & 16,33 & 11 & -5 \\
E2 & $\mathbf{6 4}$ & 29 & 23,67 & 13 & 2,33 & -3 & -19 \\
E2 & $\mathbf{5 7}$ & 22 & 16,67 & 6 & $-4,67$ & -10 & -26 \\
E2 & $\mathbf{5 4}$ & 20 & 14,67 & 4 & $-6,67$ & -12 & -28 \\
E2 & $\mathbf{5 1}$ & 16 & 10,67 & 0 & $-10,67$ & -16 & -32 \\
E2 & $\mathbf{5 0}$ & 13 & 7,67 & -3 & $-13,67$ & -19 & -35 \\
E3 & $\mathbf{9 6}$ & 80 & 74,67 & 64 & 53,33 & 48 & 32 \\
E3 & $\mathbf{5 0}$ & 39 & 35 & 26,5 & 18 & 13,75 & 1 \\
\hline
\end{tabular}

Table 3. Change of post to the unit $\mathrm{E} 2(\mathrm{k}=2)$

\begin{tabular}{cccccccc}
\hline Original site & $\begin{array}{c}\beta_{i j} \\
(\alpha=0)\end{array}$ & $\begin{array}{c}\beta_{i j} \\
(\alpha=1 / 4)\end{array}$ & $\begin{array}{c}\beta_{i j} \\
(\alpha=1 / 3)\end{array}$ & $\begin{array}{c}\beta_{i j} \\
(\alpha=1 / 2)\end{array}$ & $\begin{array}{c}\beta_{i j} \\
(\alpha=2 / 3)\end{array}$ & $\begin{array}{c}\beta_{i j} \\
(\alpha=3 / 4)\end{array}$ & $\begin{array}{c}\beta_{i j} \\
(\alpha=1)\end{array}$ \\
\hline E1 & $\mathbf{9 0}$ & 83 & 79 & 70,5 & 62 & 57,75 & 45 \\
E1 & $\mathbf{8 9}$ & 78 & 74 & 65,5 & 57 & 52,75 & 40 \\
E1 & $\mathbf{8 3}$ & 76 & 72 & 63,5 & 55 & 50,75 & 38 \\
E1 & $\mathbf{8 2}$ & 69 & 65 & 56,5 & 48 & 43,75 & 31 \\
E1 & $\mathbf{7 7}$ & 58 & 54 & 45,5 & 37 & 32,75 & 20 \\
E1 & $\mathbf{6 3}$ & 21 & 17 & 8,5 & 0 & $-4,25$ & -17 \\
E1 & $\mathbf{6 2}$ & 9 & 5 & $-3,5$ & -12 & $-16,3$ & -29 \\
E3 & $\mathbf{6 6}$ & 86 & 82 & 73,5 & 65 & 60,75 & 48 \\
E3 & $\mathbf{5 4}$ & 74 & 70 & 61,5 & 53 & 48,75 & 36 \\
E3 & $\mathbf{5 1}$ & 60 & 56 & 47,5 & 39 & 34,75 & 22 \\
E3 & $\mathbf{5 1}$ & 35.25 & 53 & 44,5 & 36 & 31,75 & 19 \\
E3 & $\mathbf{5 0}$ & 39 & 35 & 26,5 & 18 & 13,75 & 1 \\
\hline
\end{tabular}

Table 4. Change of post to the unit $\mathrm{E} 3(\mathrm{k}=3)$

\begin{tabular}{cccccccc}
\hline \multirow{2}{*}{ Original site } & $\beta_{i j}(\alpha=0)$ & $\begin{array}{c}\beta_{i j} \\
(\alpha=1 / 4)\end{array}$ & $\begin{array}{c}\beta_{i j} \\
(\alpha=1 / 3)\end{array}$ & $\begin{array}{c}\beta_{i j} \\
(\alpha=1 / 2)\end{array}$ & $\begin{array}{c}\beta_{i j} \\
(\alpha=2 / 3)\end{array}$ & $\begin{array}{c}\beta_{i j} \\
(\alpha=3 / 4)\end{array}$ & $\beta_{i j}(\alpha=1)$ \\
\hline E1 & $\mathbf{9 0}$ & 83 & 79 & 70,5 & 62 & 57,75 & 45 \\
E1 & $\mathbf{8 9}$ & 78 & 74 & 65,5 & 57 & 52,75 & 40 \\
E1 & $\mathbf{8 3}$ & 76 & 72 & 63,5 & 55 & 50,75 & 38 \\
E2 & $\mathbf{8 2}$ & 69 & 65 & 56,5 & 48 & 43,75 & 31 \\
E2 & $\mathbf{7 7}$ & 58 & 54 & 45,5 & 37 & 32,75 & 20 \\
E2 & $\mathbf{6 3}$ & 21 & 17 & 8,5 & 0 & $-4,25$ & -17 \\
\hline
\end{tabular}

Table 5. The example of average weight and capacity constraint values

\begin{tabular}{l|ccc}
\hline Average weight $\overline{\mathrm{W}}_{0}^{\mathrm{k}}$ & 96,2 & 79,72 & 114,71 \\
Capacity constraint $\mathrm{C}_{\mathrm{k}}$ & 90 & 83 & 79 \\
\hline
\end{tabular}

\subsection{Results and discussion}

The following table summarizes the main results of our test. They were obtained on a computer having CPU Pentium is $2.5 \mathrm{GHz}$ with $4 G B$ of RAM. The genetic algorithm used was encoded by the $C++$ language.

For better interpretation, the obtained numerical results are graphically visualized in the next figures. On the primary experience, we interest to show the influence of number of candidates on the generation time and convergence time. In order to do this, we take three instances composed consecutively of 29,58 and 116 candidates. In this test, the genetic algorithm is started with 40 individuals considered as the initial population size, and 50 iterations as the stopping condition applied [1]. These two parameters were experimentally justified by the figures analysis.

Other results are presented, they concern the study of the time consumed to generate the initial populations for different sizes according on number of candidates wishing to change their positions and these results are illustrated in the Figure 1. By regarding this figure, we can observe that the generation time is increased with the effective of candidates. In addition, if we elevate the number of candidates by a portion 2 or 3 , the generation time can also be assessed by the same portion. 


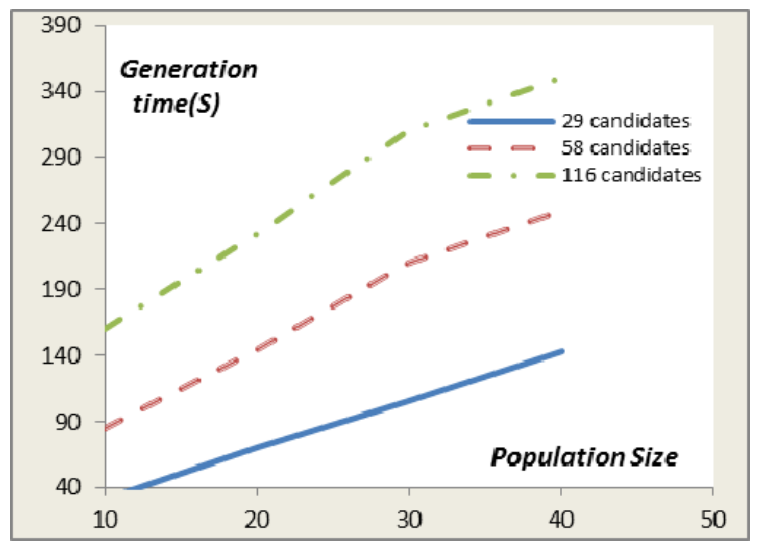

Figure 1. Generation time depending on number of individuals

The main goal of this research is to carry out investigation of the influence of one of the key GA parameters - population size (number of chromosomes) - on the algorithm performance for identification of an optimal matrix of reassignment of candidates.

On Figure 2, the precision issue the fitness function values, obtained during the 60 iterations by using a different population size: 10, 20,30 and 40 are shown. Each test consists to determine the good population size to lead to the best solution.

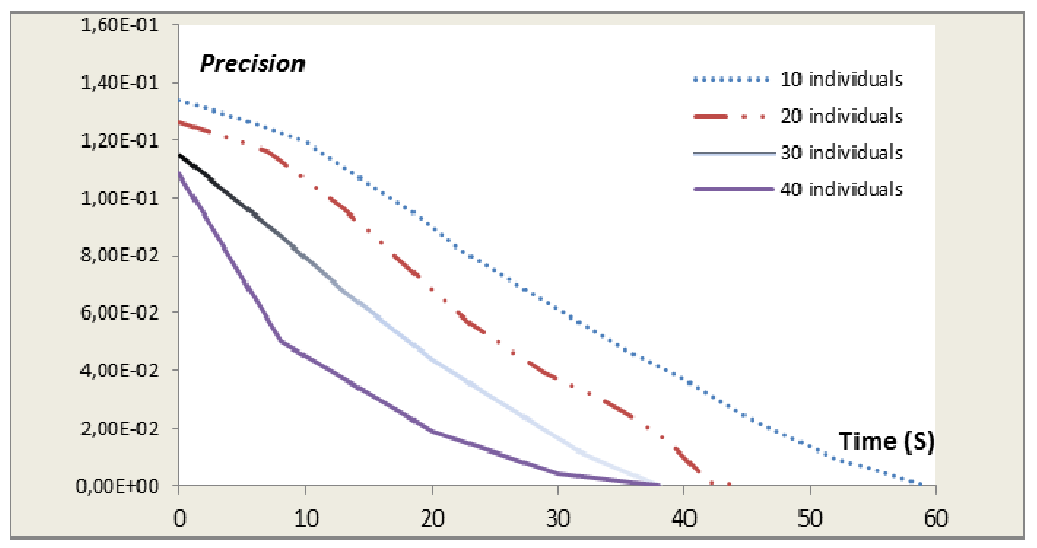

Figure 2. Precision factor depending on execution Time (Without Generation time) for an instance composed of 29 candidates

The precision factor calculated by this expression: $P=\frac{F(\operatorname{Sop} t)-F(S 0)}{F(S o p t)}$

Where Sopt is the average solution optimal obtained for 10 tests and $S 0$ is the best solution issue from the initial population. The graphical results show that the GA could not find accurate solution using small population size under 20 chromosomes. That needs at least 30 chromosomes in population for achieving a better solution of the generation time according to the number of genetic population. We can also conclude that the probability to obtain a precision factor approximately equal to 0 .

On Figure 3, the fitness function values, obtained during the 60 algorithm runs for 40 individual are shown. It can be observed in this figure that the improvement of the fitness function $(F)$ is rapid and becomes slower until the stagnation of this function. This stagnation is to show the convergence of the objective function to an optimal value $W o p t=1061.7$ for $\alpha=\frac{1}{4}$. The optimal solution $X$ obtained corresponds to global weighted matrix $\beta^{\alpha}$ that is presented as follow: 


$$
\beta^{\alpha}=\left(\begin{array}{l}
\beta^{\alpha} 1 \\
\beta^{\alpha} 2 \\
\beta^{\alpha} 3
\end{array}\right) \text { and } X=\left(\begin{array}{l}
X 1 \\
X 2 \\
X 3
\end{array}\right)
$$

With :

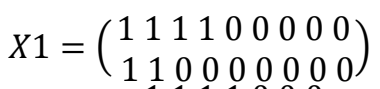

$$
\begin{aligned}
& X 2=\left(\begin{array}{lllllll}
1 & 1 & 1 & 1 & 0 & 0 & 0 \\
1 & 1 & 0 & 0 & 0 & 0 & 0
\end{array}\right) \\
& X 3=\left(\begin{array}{lll}
1 & 1 & 0 \\
1 & 1 & 0
\end{array}\right)
\end{aligned}
$$

The value 1 indicates that the employee is reassigned, however the value 0 indicates that the employee is not reassigned.

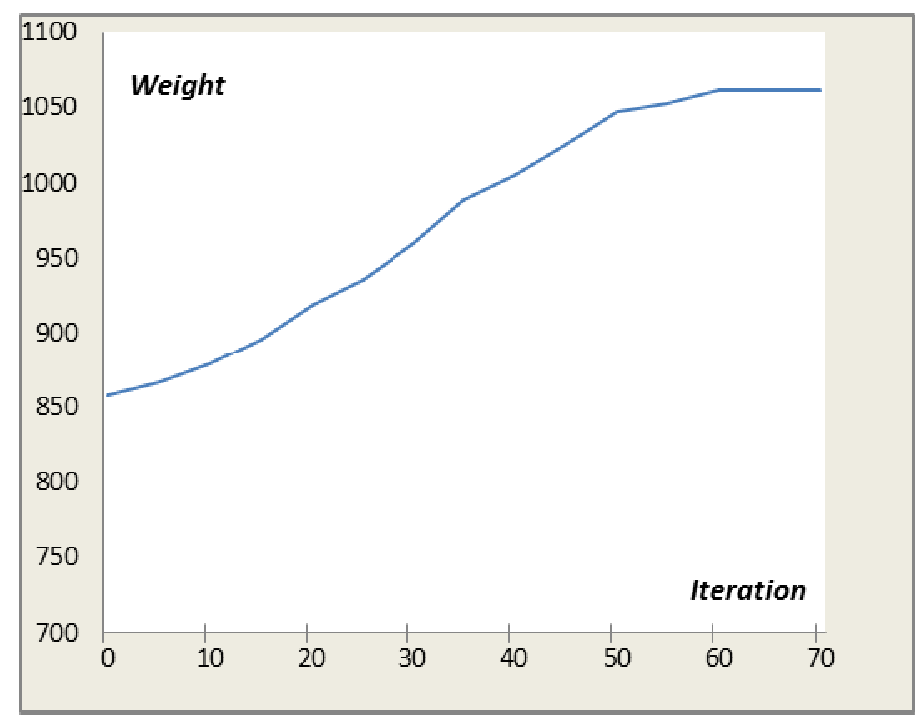

Figure 3. Variation of fitness function depending on number of iterations an instance composed of 29

$$
\text { candidates }\left(\alpha=\frac{1}{4}\right)
$$

The algorithm described above has various parameters, including the influential values strongly on its quality. Indeed, we conducted an empirical study of the adjustment of these parameters to obtain good results of performance. In this approach, we have integrated the operator uniform matrix crossover and mutation matrix (matrix HPRM) [3],[10]. These two operators are operated alternately following a so-called "Flip-Flop crossover-mutation." [3]. This implies that the mutation operator is able to be reached only in the case when the solution obtained by the crossing procedure does not satisfy all the constraints. In addition, the choice of stopping criterion based on the stabilization of the fitness function strengthens the convergence of this approach towards good solution. These new procedures also allow reduce the computation time and improves solution the solution [3].

In order to better judge the validity of our approach, we make a comparison between our approach and genetic approach used to solve the first work "A Meta-heuristically Approach of the Spatial Assignment Problem of Human Resources in Multi-sites Enterprise" [1] according to genetic operators and the stop condition. In this work we applied the maximum number of iterations as a stopping criterion which we predefined in the onset running. However, this criterion is insufficient to falling into the best solution. In addition, the application of classical crossover and mutation operators in the work [1] serves only serves to provide a solution in more time and lower quality compared to the solution obtained by our approach described in this paper.

Through a synthesis of the literature on human resource allocation problems, there is no instance targeted to numerically compare the results associated of these problems with the results obtained by our approach. Indeed, we are interested to focus on the work in treating certain term constrained assignment problem solving by genetic algorithms. The common point focuses on the use of conventional stop criteria 
based on the number of maximum iterations and secondly on the application of crossovers and mutation operators adapted and reconstructed for the problem studied, we cite for example I.Younas [18], J.Park [19] .Xilin [20], and El Moudani [21].

Table 6 shows the results corresponding to number of displacements according to $\alpha$. We see that this number can be reduced to 14 candidates when this coefficient is increased to $1 / 3$ given that 18 assigned candidates has engendered a global weight 1435 and 14 assigned candidates has engendered a global weight 948.91. In addition, these movements become not possible where $\alpha \geq 0.5$, this is can be explained by dissatisfied of objective constraint.

Table 6. Induced weight and number of possible displacement depending on tolerance factor

\begin{tabular}{ccc}
\hline Tolerance coefficient & $\begin{array}{c}\text { Number } \\
\text { of possible replacement }\end{array}$ & $\operatorname{Trace}\left(\beta^{k, \alpha} X^{k}\right)$ \\
\hline$\alpha=0$ & 18 & 1435 \\
$\alpha=1 / 4$ & 16 & 1061.7 \\
$\alpha=1 / 3$ & $14 \quad$ All displacements are interdict \\
$\alpha>=1 / 2$ & \multicolumn{2}{c}{. }
\end{tabular}

Table 7 shows the variation between $\Delta W_{k}$ (global weight) engendered by the personnel reassignment depending on tolerance coefficient $\alpha$. The numerical experiments show that increasing the gap $\Delta W_{k}$ that can be considerably as an improvement the resulting value of the objective function (average results). The results show that variation of global weight or profile $\Delta \mathrm{W}_{\mathrm{k}}$ is decreasing with $\alpha$ However, the best parameters of $\alpha$ are $\alpha=\frac{1}{4}$ and $\alpha=\frac{1}{3}$ witch conduct to improvement or equilibrium of the global weight variation $\left(\Delta \mathrm{W}_{\mathrm{k}} \geq 0\right)$ for each unit of production $E_{k}$.

Table 7. The improvement weight by the reassignment process for each unit ek depending on tolerance coefficient

\begin{tabular}{ccccc}
\hline Value of $\boldsymbol{\alpha}$ & Site & E1 (k=1) & E2 ( k=2) & E3 ( k=3) \\
\hline \multirow{4}{*}{$\alpha=0$} & $\bar{W}_{k}^{0}$ & 74 & 71 & 72 \\
& $W_{k}^{+}$ & 569 & 541 & 351 \\
& $W_{k}^{-}$ & 550 & 488 & 288 \\
& $\Delta W_{k}$ & 19 & 53 & 63 \\
& $\Delta_{k}$ & 2,17 & 4,61 & 3,50 \\
& $\bar{W}_{k}$ & 26 & 22,72 & 18,26 \\
$\alpha=1 / 4$ & $W_{k}^{+}$ & 495 & 464 & 351 \\
& $W_{k}^{-}$ & 473 & 424 & 288 \\
& $\Delta W_{k}$ & 12 & 40 & 63 \\
& $\Delta_{k}$ & 1,38 & 2,50 & 3,94 \\
& $\bar{W}_{k}$ & 25,89 & 24,59 & 23,73 \\
& $W_{k}^{+}$ & 418 & 382 & 351 \\
& $W_{k}^{-}$ & 411 & 357 & 288 \\
& $\Delta W_{k}$ & 7 & 25 & 63 \\
& $\Delta \bar{W}_{k}$ & 0.7 & 2.08 & 9 \\
& $\bar{W}_{k}^{0}$ & 29,38 & 21,92 & 18,91 \\
& & Objective constraint is not satisfied & \\
\hline
\end{tabular}

In order to study the problem of personnel reassignment by using this genetic algorithm. We used 30 individuals as population size and 50 iterations as stopping condition. We have show that the solution obtained in the reasonable time can be improved the productivity or maintain it to equilibrium productivity for each unit $E_{k}$. The parameter $\alpha$ must be verified the following condition: $\alpha<W_{\text {moy }}^{\mathrm{k}} / \overline{\mathrm{W}}_{0}^{\mathrm{k}}$ for starting our genetic algorithm in good conditions to obtain the best solution.

\section{CONCLUSION}

We presented in this paper an optimizing approach for multi constraints assignment personnel problem. This approach allow ensuring the optimal repartition of human resources to achieve the desired objective fixed by managers. We showed that this problem is very complex and it is similar proximately to Generalized Assignment Problem. For this, we used a genetic algorithm to solve this problem by the good choice of parameters genetic and tolerance coefficient. The optimal solution obtained in the reasonable time ensures at less improvement the productivity or at less conduct to equilibrium of average productivity for each unit of production. The optimal solution obtained allows validating the proposed approach. 


\section{REFERENCES}

[1] T. Said, et al., "A Meta-heuristically Approach of the Spatial Assignment Problem of Human Resources in Multisites Enterprise," International Journal of Computer Applications, vol/issue: 77(7), 2013.

[2] T. Said, et al., "A Genetic Approach for a Reassignment Problem of Human Resources under Objective Constraint," Journée scientifique en sciences appliquées JSSA'14 à Larache, Maroc, 2014.

[3] T. Said, et al., "A Hybrid Heuristic Method to Solve an Assignment Problem of Human Resource," International Review on Computers and Software (I.RE.CO.S.), vol/issue: 10(9), 2015.

[4] Haoyama and Iyetomi, "Equilibrium distribution of labor productivity," RIETI discussion paper series 12-e-041, 2012 .

[5] M. Sanghamitra and M. K. Nayak, "Optimization Model in Human Resource Management for Job Allocation in ICT Project," International Journal of the Computer, the Internet and Management, vol. 19. Pp. 21 -27, 2011.

[6] J. K. Hao, "Métaheuristiques pour l'optimisation combinatoire et l'affectation sous contraintes," Revue d'Intelligence Artificielle, 1999.

[7] Frits C. R., "Spieksma Multi Index Assignment Problems: Complexity, Approximation," Applications Nonlinear Assignment Problems Combinatorial Optimization, vol. 7, pp. 1-12, 2000.

[8] O. Abdoun and J. Abouchabaka, "A Comparative Study of Adaptive Crossover Operators for Genetic Algorithms to Resolve the Travelling Salesman Problem," International Journal of Computer Applications, vol/issue: 31(11).

[9] O. Abdoun, et al., "Analyzing the Performance of Mutation Operators to Solve the Traveling Salesman Problem," International Journal of Emerging Sciences, vol/issue: 2(1).

[10] O. Abdoun, et al., "Hybridizing PSM and RSM Operator for Solving NP-Complete Problems: Application to Traveling Salesman Problem," International Journal of Computer Science Issues, vol/issue: 9(1).

[11] M. Yagiura, et al., "An ejection chain approach for the generalized assignment problem," INFORMS Journal on Computing, vol. 16, pp. 131-151, 2004.

[12] D. G. Cattrysse, "A survey of algorithms for the generalize assignment problem," European Journal of Operational Research, vol. 60, pp. 260-272, 1992.

[13] Holland J. H., "Adaptation in Natural and Artificial Systems," University of Michigan Press (Ann Arbor), 1975.

[14] D. E. Goldberg, "Genetic Algorithms in Search," Optimization and Machine Learning, Addison-Wesley, Reading, MA, 1989.

[15] L. Davis, "Genetic Algorithm and Simulated Annealling," San Francisco, Morgan Kaufmann, 1987.

[16] S. W. Mahfoud, "Niching methods for genetic algorithms," Ph.D. dissertation, Univ. Illinois at Urbana-Champaign, Illinois Genetic Algorithm Lab., Urbana, IL, 1995.

[17] Z. Michalewicz, "Genetic algorithms and data structures: evolution programs,” Berlin, Springer, 1992.

[18] I. Younas, et al., "Efficient genetic algorithms for optimal assignment of tasks to teams of agents," IEEE Symposium on Computational Intelligence in Scheduling, Paris, France, April 2011.

[19] J. Park, et al., "Practical Human Resource Allocation in Software Projects Using Genetic Algorithm," SEKE, pp. 688-694, 2014.

[20] Z. Xilin and L. Shiming, "Optimization Model of Higher Education Resources Allocation Based on Genetic Algorithm," Management Science and Engineering, vol/issue: 7(3), pp. 76-80, 2013. DOI:10.3968/j.mse.1913035X20130703.2622

[21] E. Moudani W., et al., "A bi-criterion Approach for the Airlines Crew Rostering Problem," LAAS report 00428, October 2000.

\section{BIOGRAPHIES OF AUTHORS}

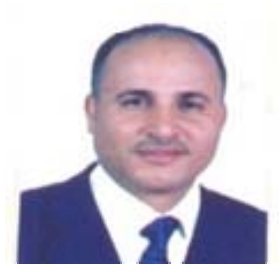

S. Tkatek, he recived his Diploma of Advanced Graduate Studies in physics, 2000 from faculty of science, Ibn tofail university, Kenitra, Morocco. $\mathrm{He}$ is a $\mathrm{PhD}$ Candidate currently. Email: saidtkinfo@yahoo.fr

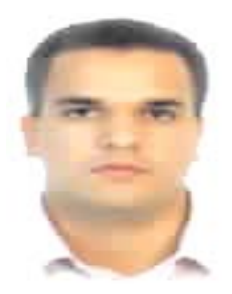

O. Abdoun received his MS degree from Ibn Tofail University in Mathematics and computer science. He received his $\mathrm{PhD}$ degree from Ibn Tofail University in Comuter Science. Dr. Abdoun is currently a professor at the department of computer science, Polydisciplinary Faculty, Abdelmalk Essaadi University, Larache, Morocco. Email: abdoun@fpl.ma 


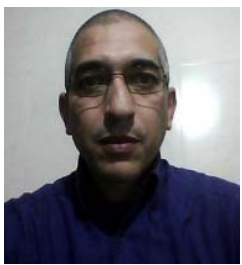

J. Abouchabaka received his MS degree from Mohamed V University in Applied Mathematics. He received his $\mathrm{PhD}$ degree from Mohamed V University in Applied Mathematics. Dr. Abouchabaka has served as a faculty member in the computer science department in Ibn Tofail University, Kenitra, Morocco. Email: abouchabaka-jaafar@univ-ibntofail.ac.ma

N. Rafalia received his MS degree from Mohamed V University in Computer Science. She received his Habitation thesis from Ibn Tofail University. Dr. Rafalia is currently a Professor in the informatics department in the faculty of sciences, Kenitra, Morocco. Email: arafalia@yahoo.com 